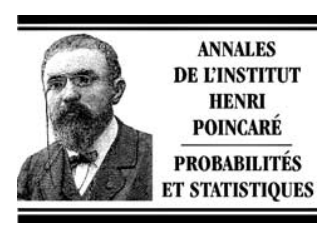

www.elsevier.com/locate/anihpb

\title{
Approximations of effective coefficients in stochastic homogenization
}

\author{
Alain Bourgeat ${ }^{\mathrm{a}}$, Andrey Piatnitski ${ }^{\mathrm{b}, \mathrm{c}}$ \\ ${ }^{a}$ Université Lyon 1, MCS - bât.ISTIL, 15, bld Latarjet, 69622 Villeurbanne Cedex, France \\ ${ }^{\mathrm{b}}$ Narvik University College, Postboks 385, 8505 Narvik, Norway \\ ${ }^{\mathrm{c}}$ P.N. Lebedev Physical Institute of RAS, Leninski pr., 53, Moscow 117924, Russia
}

Received 3 September 2002; received in revised form 24 June 2003; accepted 17 July 2003

\begin{abstract}
This note deals with localized approximations of homogenized coefficients of second order divergence form elliptic operators with random statistically homogeneous coefficients, by means of "periodization" and other "cut-off" procedures. For instance in the case of periodic approximation, we consider a cubic sample $[0, \rho]^{d}$ of the random medium, extend it periodically in $\mathbb{R}^{d}$ and use the effective coefficients of the obtained periodic operators as an approximation of the effective coefficients of the original random operator. It is shown that this approximation converges a.s., as $\rho \rightarrow \infty$, and gives back the effective coefficients of the original random operator. Moreover, under additional mixing conditions on the coefficients, the rate of convergence can be estimated by some negative power of $\rho$ which only depends on the dimension, the ellipticity constant and the rate of decay of the mixing coefficients. Similar results are established for approximations in terms of appropriate Dirichlet and Neumann problems localized in a cubic sample $[0, \rho]^{d}$.
\end{abstract}

(C) 2004 Elsevier SAS. All rights reserved.

\section{Résumé}

Nous étudions différentes procédures de périodisation ou troncature pour approcher les coefficients effectifs d'un opérateur elliptique du second ordre à coefficients aléatoires stationnaires. Considérons par exemple la restriction d'un environnement aléatoire à un cube $[0, \rho]^{d}$ et son prolongement périodique à $\mathbb{R}^{d}$ tout entier. Nous montrons qu'alors, pour presque toute réalisation de l'environnement aléatoire, les coefficients homogénéisés dans l'approximation périodique convergent quand $\rho \rightarrow \infty$ vers les coefficients effectifs de l'opérateur initial. Sous des hypothèses de mélange nous prouvons des bornes sur la vitesse de convergence de la forme $\rho^{-\alpha}$ où $\alpha>0$ ne dépend que de la dimension, la constante d'ellipticité et du taux de mélange. Nous obtenons aussi des résultats similaires pour des approximations basées sur des problèmes de Neumann ou de Dirichlet, localisées dans un cube $[0, \rho]^{d}$.

(C) 2004 Elsevier SAS. All rights reserved.

MSC: 35K20; 35Q35; 35R60

Keywords: Random operator; Volume averaging; Homogenization

E-mail addresses: bourgeat@mcs.univ-lyon1.fr (A. Bourgeat), andrey@sci.lebedev.ru (A. Piatnitski). 


\section{Introduction}

The main goal of this work is to provide a rigorous mathematical justification for the convergence and to give, whenever possible, estimates for the rate of convergence of the various localization methods used in engineering literature to approximate the effective tensor of random stationary media. The question on how to get the effective parameters characterizing the constitutive equation in randomly heterogeneous media has been a subject of concern in many scientific fields including solid and fluid mechanics, hydrogeology or thermics. It was a subject of interest for engineers, in connection with applications in composite media [11,17,21], in oil recovery [9,1], in underground pollutant transport [2,12]. All these practical methods are based on computing the average of either the energy or the flux on a sufficiently big volume, called Representative Elementary Volume (or REV), with some boundary conditions like for instance periodic or Dirichlet or Neumann boundary conditions. The same kind of averaging on a finite volume is also systematically used, in the so called Volume Averaging method (see for instance [5]), for deriving theoretical scaled up models from microscopic phenomenological descriptions. Clearly all these approximated effective characteristics, obtained from a finite volume, are in general still random. And behind all these computations there is no indication on how this averaging, on a finite part of only one realization, with one of the mentioned boundary conditions, is related to the effective tensor given by the rigorous methods of stochastic homogenization.

First mathematical results on stochastic homogenization of linear second order divergence form elliptic operators were obtained in [14] and in [20]. Later on other stochastic models have been studied and new methods have been developed in the works $[3,6,7,13,15,18,21]$ and many others.

In these works it was proved that the homogenization result holds almost surely and that the effective operator can be found in terms of a solution of a certain auxiliary problem. Since this auxiliary problem is stated in an abstract probability space, this formal mathematical technique does not give any practical recipe for constructing or approximating the effective characteristics. On the contrary to the random case, in the case of periodic media there are many efficient numerical homogenization procedures.

One of the important aspect of homogenization theory, both in applications and from the theoretical point of view, is the rate of convergence of homogenization procedure. This question is getting extremely difficult in the random case. An important result was obtained in [24], where boundary value problems for a second order divergence form operator were studied and, under proper mixing condition, polynomial bounds for the convergence rate of boundary value problems solutions were achieved. For a system of equations only logarithmic bounds have been obtained, see [22].

The estimate of discrepancy in various cut-off approximations procedures is another important problem. It seems, according to the authors present knowledge, that there is still no rigorous result on that subject. In the present paper we prove the convergence and estimate the rate of convergence for the typical "practical homogenization procedures" applied to random second order uniformly elliptic operators with statistically homogeneous ergodic coefficients.

The first section is devoted to a complete definition of the random media and to the precise description of the effective coefficients obtained by homogenization as it appears in [14,20], and in [13]. Then, in the same section, we recall the three widely used ways of approximating the effective tensors by averaging the operator on an elementary volume with different boundary conditions.

In Section 2 we study the accuracy of all these approximations and prove their almost sure convergence, as the elementary volume tends to infinity, to the effective characteristics obtained by homogenization of the original stochastic operator. These results give also rigorous mathematical justification for most of the approximation procedures used in the engineering literature [23]; see Remark 2 below.

It should be noted that the convergence of approximations by periodization has been studied by probabilistic methods in the recent work [19]. However, our approach gives rise to a shorter proof and allows us, under certain conditions, to estimate the rate of convergence. 
Finally, in the last section, under the additional uniform mixing conditions on coefficients similar to those in [24], we estimate the rates of convergence of these approximations and show that the corresponding bounds only depend on the ellipticity constant in the original problem, the space dimension, and the rate of decay of the uniform mixing coefficients. To this end we penalize the original operator by adding a small positive potential and introduce "effective auxiliary characteristics" associated to this penalized operator. These effective auxiliary characteristics are then approximated by applying to the penalized operator one of the mentioned cut-off procedures. Finally we show, that there is always a properly chosen potential depending on the sample size, such that all the discrepancies involved admit polynomial bounds.

\section{Definition of random media and approximation models}

We begin by giving the general assumptions and definitions.

Let $(\Omega, \mathcal{F}, \mathbf{P})$ be a standard probability space, and assume that a $d$-dimensional dynamical system $T_{z}, z \in \mathbb{R}^{d}$, is given on $\Omega$, i.e. a family of invertible measurable maps $T_{z}: \Omega \rightarrow \Omega, z \in \mathbb{R}^{d}$, such that

$-T_{x+y}=T_{x} T_{y}, T_{0}=\mathrm{Id}$

- $T_{x}$ preserves the measure $\mathbf{P}$ that is $\mathbf{P}\left\{\left(T_{x}\right)^{-1}(\mathcal{A})\right\}=\mathbf{P}\{\mathcal{A}\}$ for any $\mathcal{A} \in \mathcal{F}$ and any $x \in \mathbb{R}^{d}$;

- $T_{x}$ is a measurable mapping from $\mathbb{R}^{d} \times \Omega$ to $\Omega$, where $\mathbb{R}^{d} \times \Omega$ is equipped with the product $\sigma$-algebra $\mathcal{B} \times \mathcal{F}$ and $\mathcal{B}$ is the Borel $\sigma$-algebra in $\mathbb{R}^{d}$.

In the presence of such a dynamical system, a wide class of statistically homogeneous random fields can be introduced as follows: for an arbitrary random variable $f=f(\omega)$ we define $f(z, \omega) \equiv f\left(T_{z} \omega\right)$. It is then easy to check that $f(z, \omega)$ is a statistically homogeneous random field. In this work we suppose that the coefficients of random operators are defined in terms of a dynamical system $T_{z}$.

Next we introduce the notion of ergodicity. By definition, a subset $\mathcal{A} \in \mathcal{F}$ is invariant if $T_{z}(\mathcal{A})=\mathcal{A}$ for any $z \in \mathbb{R}^{d}$. A dynamical system $T_{z}$ is said to be ergodic if for any invariant set $\mathcal{A} \in \mathcal{F}$ we have either $\mathbf{P}(\mathcal{A})=1$ or $\mathbf{P}(\mathcal{A})=0$.

Although the homogenization result remains valid for nonergodic operators, the ergodicity assumption allows us to simplify the calculations; in this connection, if the opposite is not indicated explicitly, we suppose in the following sections that the dynamical system $T_{z}$ is ergodic.

We recall now the main results on homogenization of random operators.

\subsection{Random operators}

For a given matrix-function $a_{i j}=a_{i j}(\omega)$ such that

$$
\begin{aligned}
& a_{i j}(\omega) \eta_{i} \eta_{j} \geqslant \lambda|\eta|^{2}, \quad \eta \in \mathbb{R}^{d}, \lambda>0, \\
& a_{i j}(\omega) \leqslant \lambda^{-1},
\end{aligned}
$$

we define the following family of operators

$$
A_{\varepsilon}=\frac{\partial}{\partial x_{i}} a_{i j}\left(T_{x / \varepsilon}(\omega)\right) \frac{\partial}{\partial x_{j}} .
$$

It is then well known from the works [14], [20] (see also [13] and the quoted literature there) that the family $A_{\varepsilon}$ admits almost surely (a.s.), as $\varepsilon \downarrow 0$, a homogenized limit also called the "effective operator":

$$
\hat{A}=\hat{a}_{i j} \frac{\partial}{\partial x_{i}} \frac{\partial}{\partial x_{j}},
$$


where $\hat{a}=\left\{\hat{a}_{i j}\right\}$ is a constant matrix. By definition, this means that for any Lipschitz bounded domain $Q \subset \mathbb{R}^{d}$ and any $f \in H^{-1}(Q)$, a solution to the problem

$$
A_{\varepsilon} u^{\varepsilon}(x)=f(x) \quad \text { in } Q,\left.\quad u^{\varepsilon}(x)\right|_{\partial Q}=0
$$

converges a.s., as $\varepsilon \downarrow 0$, to a solution $u^{0}$ of the limit problem

$$
\hat{A} u^{0}(x)=f(x) \quad \text { in } Q,\left.\quad u^{0}(x)\right|_{\partial Q}=0 .
$$

The matrix $\left\{\hat{a}_{i j}\right\}$ is known to be positive definite so that problem (5) has a unique solution.

In the ergodic case, this constant matrix $\left\{\hat{a}_{i j}\right\}$ is nonrandom. Without ergodicity assumption, the homogenization result still holds with the only difference that the effective coefficients $\hat{a}_{i j}$ are no more deterministic, but measurable with respect to the $\sigma$-algebra of invariant sets

$$
\mathcal{F}_{\text {inv }}=\left\{\mathcal{A} \in \mathcal{F} \mid T_{z}(\mathcal{A})=\mathcal{A} \text { for all } z \in \mathbb{R}^{d}\right\} .
$$

\subsection{Homogenization procedure}

For the reader's convenience we outline briefly the homogenization procedure in the random ergodic case. Let $U_{z}$ be a $d$-parameter strongly continuous group of unitary operators in $L^{2}(\Omega)=L^{2}(\Omega, \mathcal{F}, \mathbf{P})$, associated with $T_{z}$ :

$$
\left(U_{z} f\right)(\omega)=f\left(T_{z} \omega\right), \quad f \in L^{2}(\Omega) .
$$

We suppose that $L^{2}(\Omega)$ is separable. Denote by $\partial_{\omega}^{j}$ the generator of $U_{z}$ along $j$ th coordinate direction, i.e.

$$
\partial_{\omega}^{j}=\lim _{\delta \rightarrow 0} \delta^{-1}\left(f\left(T_{\delta e_{j}} \omega\right)-f(\omega)\right) .
$$

The domains $\mathcal{D}_{j}$ of $\partial_{\omega}^{j}$ are dense in $L^{2}(\Omega)$, and the intersection $\mathcal{D}=\bigcap_{j=1}^{d} \mathcal{D}_{j}$ is also dense in $L^{2}(\Omega)$ (see [8] Lemma VIII.1.8 and Ex. VIII.3.10, and [6]).

For $f \in \mathcal{D}$ and $\mathbf{g}=\left(g_{1}, \ldots, g_{d}\right) \in(\mathcal{D})^{d}$ we define

$$
\nabla_{\omega} f=\left(\partial_{\omega}^{1} f, \ldots, \partial_{\omega}^{d} f\right) ; \quad \operatorname{div}_{\omega} \mathbf{g}=\sum_{j=1}^{d} \partial_{\omega}^{j} g_{j},
$$

and then introduce the following subspaces of $\left(L^{2}(\Omega)\right)^{d}$

$$
\begin{aligned}
& L_{\text {pot }}^{2}(\Omega)=\overline{\left\{\mathbf{v} \in\left(L^{2}(\Omega)\right)^{d} \mid \mathbf{v}=\nabla_{\omega} f \text { for some } f \in \mathcal{D}\right\}}, \\
& L_{\text {sol }}^{2}(\Omega)=\overline{\left\{\mathbf{v} \in(\mathcal{D})^{d} \mid \operatorname{div}_{\omega} \mathbf{v}=0\right\}},
\end{aligned}
$$

where the overline symbol means the closure in $\left(L^{2}(\Omega)\right)^{d}$.

The effective coefficients could be then obtained from solutions of the following family of auxiliary problems:

$$
\text { given } \eta \in \mathbb{R}^{d} \text {, find } \mathbf{v}_{\eta} \in L_{\text {pot }}^{2}(\Omega) \quad \text { such that } a(\omega)\left(\mathbf{v}_{\eta}+\eta\right) \in L_{\text {sol }}^{2}(\Omega) \text {. }
$$

For any vector $\eta$ in $\mathbb{R}^{d}$ this problem is well-posed and has a unique solution (see, for instance, [13]); it is also clear, that $\mathbf{v}_{\eta}$ is a linear function of $\eta$. The coefficients $\hat{a}_{i j}$ of effective operator (3) or, briefly, effective coefficients are now computed as follows:

$$
\hat{a} \eta=\int_{\Omega} a(\omega)\left(\mathbf{v}_{\eta}(\omega)+\eta\right) \mathbf{P}(d \omega) .
$$

If the medium is not ergodic, then $\left\{\hat{a}_{i j}\right\}$ is a random matrix that can be found in terms of auxiliary problem (6) as follows:

$$
\hat{a}(\omega) \eta=\mathbf{E}\left\{a(\omega)\left(\eta+\mathbf{v}_{\eta}(\omega)\right) \mid \mathcal{F}_{\text {inv }}\right\} .
$$


According to the Birkhoff theorem, both in ergodic and nonergodic cases, $\hat{a}$ can be approximated by spatial averages:

$$
\hat{a}=\lim _{\rho \rightarrow \infty} \rho^{-d} \int_{[0, \rho]^{d}} a\left(T_{z} \omega\right)\left(\eta+\mathbf{v}_{\eta}\left(T_{z}(\omega)\right)\right) d z .
$$

\subsection{Approximation models}

Since auxiliary problem (6), used for defining the effective coefficients, is stated in an abstract space, it does not allow any natural direct approximation procedures. Due to this reason, in applications the cut-off technique is used, and the solution of problem (6) is usually approximated by a solution of problems stated on a torus, or in a bounded domain with Dirichlet, Neumann or appropriate mixed boundary conditions.

We consider here three typical approximation models used in practice, and for all of them we use a cubic sample $[0, \rho]^{d}$ denoted by $S_{\rho}$. However, it should be noted that the cubic sample is chosen just for notations convenience. In the case of approximations based on boundary value problems in cut-off domains, with appropriate Dirichlet, Neumann or mixed boundary conditions, all our claims remain valid if we use homothetic dilatations of a general regular bounded domain instead of a cubic sample.

\subsubsection{Periodic approximation}

The coefficients $a_{i j}\left(T_{z} \omega\right)$ are first restricted onto the cube $S_{\rho}$ and then extended from this cube to the whole space $\mathbb{R}^{d}$ periodically with period $\rho$ in each coordinate direction so that

$$
a_{\mathrm{per}}^{\rho}(z, \omega)=a\left(T_{z\left(\bmod S_{\rho}\right)} \omega\right) .
$$

For each $\omega \in \Omega$ the family of operators

$$
A_{\varepsilon}^{\rho}=\frac{\partial}{\partial x_{i}}\left(a_{\mathrm{per}, i j}^{\rho}\left(\frac{x}{\varepsilon}, \omega\right)\right) \frac{\partial}{\partial x_{j}}
$$

has periodic coefficients and thus can be homogenized in a standard way (see, for instance, [4]). The effective operator $\tilde{A}^{\rho}$ has constant coefficients $\tilde{a}_{i j}^{\rho}$, however, since the field $a_{\mathrm{per}}^{\rho}(z, \omega)$ is not ergodic any more, the coefficients could possibly not be deterministic.

Let us recall that the matrix $\left\{\tilde{a}_{i j}^{\rho}\right\}$ can be found in terms of a solution of the following cell problem:

$$
\begin{aligned}
& \text { for any } \eta \in \mathbb{R}^{d} \text { find } \chi_{\eta}^{\rho} \in H_{\mathrm{per}}^{1}\left(S_{\rho}\right) \text { such that } \\
& -\operatorname{div}\left(a_{\mathrm{per}}^{\rho}(z, \omega)\left(\nabla \chi_{\eta}^{\rho}+\eta\right)\right)=0 \quad \text { in } \mathbb{R}^{d},
\end{aligned}
$$

where $H_{\mathrm{per}}^{1}$ is the space of $[0, \rho]^{d}$-periodic $H_{\mathrm{loc}}^{1}$-functions. The effective matrix for this periodic operator is then given by

$$
\tilde{a}^{\rho} \eta=\rho^{-d} \int_{S_{\rho}} a(z, \omega)\left(\nabla \chi_{\eta}^{\rho}+\eta\right) d z, \quad \forall \eta \in \mathbb{R}^{d} .
$$

Our aim is to show that $\tilde{a}^{\rho}$ approaches $\hat{a}$, a.s., as $\rho \rightarrow \infty$.

\subsubsection{Approximation by Dirichlet problem}

In this approximation model we make a truncation on a sample $S_{\rho}$ and impose linear Dirichlet boundary conditions on $\partial S_{\rho}$; namely we consider the equations

$$
-\operatorname{div}\left(a(z, \omega)\left(\nabla w_{\eta}^{\rho}+\eta\right)\right)=0 \quad \text { in } S_{\rho}
$$


with the linear Dirichlet boundary conditions

$$
\left.\left(w_{\eta}^{\rho}(z)+\eta \cdot z\right)\right|_{\partial S_{\rho}}=\eta \cdot z, \quad \forall \eta \in \mathbb{R}^{d} .
$$

The approximate effective coefficients are now defined $\forall \eta \in \mathbb{R}^{d}$, by

$$
\bar{a}^{\rho} \eta=\rho^{-d} \int_{S_{\rho}} a(z, \omega)\left(\nabla w_{\eta}^{\rho}+\eta\right) d z .
$$

Remark 1. It is interesting to notice that in [18] the a.s. limit of $\bar{a}^{\rho}$, as $\rho \rightarrow \infty$, was taken as a definition of homogenized matrix.

\subsubsection{Approximation by Neumann problem}

In this model we make a truncation on a sample and impose Neumann boundary conditions; namely we consider the following problems in $S_{\rho}$

$$
\begin{aligned}
& -\operatorname{div}\left(a(z, \omega)\left(\nabla \psi_{\eta}^{\rho}+\eta\right)\right)=0 \quad \text { in } S_{\rho}, \\
& \frac{\partial}{\partial v}\left(\psi_{\eta}^{\rho}(z)+\eta \cdot z\right)=\eta \cdot v \quad \text { on } \partial S_{\rho}, \quad \forall \eta \in \mathbb{R}^{d},
\end{aligned}
$$

where $v$ is the external conormal to $S_{\rho}$. Then, the effective matrix approximation is computed as follows

$$
\check{a}^{\rho} \eta=\rho^{-d} \int_{S_{\rho}} a(z, \omega)\left(\nabla \psi_{\eta}^{\rho}+\eta\right) d z, \quad \forall \eta \in \mathbb{R}^{d} .
$$

\section{Convergence results}

In this section we are going to show that the three approximations $\tilde{a}^{\rho}, \bar{a}^{\rho}$ and $\check{a}^{\rho}$ introduced in the preceding section, converge a.s., as $\rho \rightarrow \infty$, to the effective coefficients $\hat{a}$ obtained by stochastic homogenization of the original random operator (2) as was described in Section 1.

We begin by studying the first model based on Periodic Approximation.

Theorem 1. Let $\tilde{a}^{\rho}$ be the effective matrix obtained in (9) and (10) by Periodic Approximation method, then the following limit relation holds

$$
\lim _{\rho \rightarrow \infty}\left\{\tilde{a}_{i j}^{\rho}\right\}=\left\{\hat{a}_{i j}\right\} \quad \text { a.s. }
$$

Proof. Consider in the unit cube $S_{1}=[0,1]^{d}$ an auxiliary problem

$$
\begin{aligned}
& -\operatorname{div}\left(a_{\mathrm{per}}^{\rho}(\rho x, \omega) \nabla u^{\rho}\right)=f(x), \quad x \in S_{1}, \\
& \left.u^{\rho}\right|_{\partial S_{1}}=0 .
\end{aligned}
$$

Since by the definition of $a_{\mathrm{per}}^{\rho}$, we have $a_{\mathrm{per}}^{\rho}(\rho x, \omega)=a(\rho x, \omega)$ for $x \in S_{1}$, this problem is a particular case of problem (4) with $\varepsilon=1 / \rho$ and $S_{1}=Q$. Thus the homogenization result (5) applies, and the effective operator obtained by passage to the limit, as $\rho \rightarrow \infty$, in (16), coincides with $\hat{A}$.

It is convenient to rescale the variables in the cell problem (9) so that the rescaled equation is stated in the unit cube $S_{1}$. Denote $\chi_{0, \eta}^{\rho}(x)=\frac{1}{\rho} \chi_{\eta}^{\rho}(\rho x)$. In the coordinates $x=z / \rho$ problem (9) reads

$$
-\operatorname{div}\left(a_{\mathrm{per}}^{\rho}(\rho x, \omega)\left(\nabla \chi_{0, \eta}^{\rho}+\eta\right)\right)=0 \quad \text { in } \mathbb{R}^{d},
$$


where $\chi_{0, \eta}^{\rho}(x)$ is a $[0,1]^{d}$-periodic function. Denote $\chi_{0, j}^{\rho}=\chi_{0, e_{j}}^{\rho}$, where $\chi_{0, e_{j}}^{\rho}$ is a solution of (17) related to the $j$ th coordinate vector $\eta=e_{j}$ in $\mathbb{R}^{d}$, and let $\chi_{0}^{\rho}$ be the vector-function $\left(\chi_{0,1}^{\rho}, \ldots, \chi_{0, d}^{\rho}\right)$. The solution $\chi_{0}^{\rho}$ is unique up to an additive constant vector. In order to fix the choice of $\chi_{0}^{\rho}$, we assume that $\int_{S_{1}} \chi_{0}^{\rho}(x) d x=0$. A priori estimates for (17) are straightforward. Indeed, multiplying (17) by $\chi_{0, \eta}^{\rho}$, integrating by parts and applying the Poincaré inequality, we get

$$
\left\|\chi_{0}^{\rho}\right\|_{\left(H^{1}\left(S_{1}\right)\right)^{d}} \leqslant C \text {. }
$$

Hence, there is a subsequence of $\chi_{0}^{\rho}$ that converges weakly in $\left(H_{\text {per }}^{1}\left(S_{1}\right)\right)^{d}$ as $\rho \rightarrow \infty$. If we denote the limit function $\chi_{0}^{\infty}=\chi_{0}^{\infty}(x)$, then, by Theorem 5.2 in [13], $\chi_{0}^{\infty}$ satisfies the equation

$$
-\operatorname{div}\left(\hat{a}\left(\nabla \chi_{0}^{\infty}+I\right)\right)=0 \quad \text { in } S_{1},
$$

this equation can be easily solved explicitly. Its only zero average periodic solution is $\chi_{0}^{\infty}=0$. Therefore, the whole sequence $\chi_{0}^{\rho}$ converges a.s., as $\rho \rightarrow \infty$. Moreover, by the same result in [13], the fluxes also converge a.s., that is

$$
a(\rho x, \omega)\left(\nabla \chi_{0}^{\rho}+I\right) \rightarrow \hat{a}\left(\nabla \chi_{0}^{\infty}+I\right)=\hat{a} \quad \text { in }\left(L^{2}\left(S_{1}\right)\right)^{d^{2}} \text { weakly }
$$

Integrating the last relation over $S_{1}$ and taking into account formula (10) gives

$$
\tilde{a}^{\rho}=\int_{S_{1}} a(\rho x, \omega)\left(\nabla \chi_{0}^{\rho}+I\right) d x \underset{\rho \rightarrow \infty}{\longrightarrow} \int_{S_{1}} \hat{a} d x=\hat{a} .
$$

This completes the proof.

We proceed with the second model.

Theorem 2. Let $\left\{\bar{a}_{i j}^{\rho}\right\}$ be the approximate effective coefficients, obtained by Dirichlet problem Approximation, as defined in (13). Then $\left\{\bar{a}_{i j}^{\rho}\right\}$ converges a.s., as $\rho \rightarrow \infty$, to the matrix $\hat{a}$.

Proof. The proof is similar to that of the preceding theorem. We introduce $w_{0, \eta}^{\rho}(x)=\frac{1}{\rho} w_{\eta}^{\rho}(\rho x)$. In the rescaled coordinates $x=z / \rho$ problem (11)-(12) takes the form

$$
\begin{aligned}
& -\operatorname{div}\left(a(\rho x, \omega)\left(\nabla w_{0, \eta}^{\rho}+\eta\right)\right)=0 \quad \text { in } S_{1}, \\
& w_{0, \eta}^{\rho} \mid \partial S_{1}=0 .
\end{aligned}
$$

Denote $w_{0, j}^{\rho}=w_{0, e_{j}}^{\rho}$ and $\mathbf{w}_{0}^{\rho}=\left(w_{0,1}^{\rho}, \ldots, w_{0, d}^{\rho}\right)$. The estimate $\left\|\mathbf{w}_{0}^{\rho}\right\|_{\left(H^{1}\left(S_{1}\right)\right)^{d}} \leqslant C$ is evident. Let $\mathbf{w}_{0}^{\infty}$ be the limit of a weakly convergent subsequence of $\mathbf{w}_{0}^{\rho}$. Then, by Theorem 5.2 in [13], $\mathbf{w}_{0}^{\infty}$ satisfies the equation

$$
\begin{aligned}
& -\operatorname{div}\left(\hat{a}\left(\nabla \mathbf{w}_{0}^{\infty}+I\right)\right)=0 \quad \text { in } S_{1}, \\
& \left.\mathbf{w}_{0}^{\infty}\right|_{\partial S_{1}}=0,
\end{aligned}
$$

and, a.s.

$$
a(\rho x, \omega)\left(\nabla \mathbf{w}_{0}^{\rho}+I\right) \rightarrow \hat{a}\left(\nabla \mathbf{w}_{0}^{\infty}+I\right) \quad \text { in }\left(L^{2}\left(S_{1}\right)\right)^{d^{2}} \text { weakly. }
$$

Clearly, $\mathbf{w}_{0}^{\infty}=0$, therefore,

$$
\bar{a}^{\rho}=\int_{S_{1}} a(\rho x, \omega)\left(\nabla \mathbf{w}_{0}^{\rho}+I\right) d x \underset{\rho \rightarrow \infty}{\longrightarrow} \int_{S_{1}} \hat{a}\left(\nabla \mathbf{w}^{\infty}+I\right) d x=\hat{a},
$$

and the desired statement is proved. 
We study the third model by means of exactly the same procedure, and the following result holds.

Theorem 3. Let $\left\{\breve{a}_{i j}^{\rho}\right\}$ be the approximate effective coefficients, obtained by Neumann problem Approximation, as defined in (15). Then $\left\{\check{a}_{i j}^{\rho}\right\}$ converges a.s., as $\rho \rightarrow \infty$, to the matrix $\hat{a}$.

\section{Estimates for the rate of convergence}

We study the rate of convergence in the three models of Section 1 under the assumption that the random fields $a(z, \omega)$ satisfies uniform mixing condition. Our analysis relies essentially on the results obtained in [24].

For the reader's convenience we recall the definition of uniform mixing condition.

Given a statistically homogeneous random field $\xi(z, \omega)$ in $\mathbb{R}^{d}$, we denote $\mathcal{F}_{A}$ the $\sigma$-algebra $\sigma\{\xi(z), z \in A\}$. The function

$$
\alpha(s)=\sup _{\substack{A, B \in \mathbb{R}^{d} \\ \operatorname{dist}(A, B) \geqslant s}} \sup _{\mathcal{A} \in \mathcal{F}_{A}, \mathcal{B} \in \mathcal{F}_{B}}|\mathbf{P}(\mathcal{A} \cap \mathcal{B})-\mathbf{P}(\mathcal{A}) \mathbf{P}(\mathcal{B})|
$$

is said to be the uniform mixing coefficient of $\xi$.

In what follows we denote by $\alpha(s)$ the uniform mixing coefficient of the random field $a(z, \omega)=a\left(T_{z} \omega\right)$, and suppose that $\alpha(s)$ satisfies the inequality

$$
\alpha(s) \leqslant c(1+s)^{-\theta} \quad \forall s>0,
$$

for some $\theta>0$.

Let $\mathbf{v}^{\kappa}(z, \omega)$ be a solution of the following "penalized" equation in $\mathbb{R}^{d}$ :

$$
-\operatorname{div}\left(a(z, \omega) \nabla \mathbf{v}^{\kappa}\right)+\kappa \mathbf{v}^{\kappa}=\operatorname{div} a(z, \omega),
$$

with $\kappa>0$. For each positive $\kappa$ this equation has a unique solution $\mathbf{v}^{\kappa} \in\left(H_{\mathrm{loc}}^{1}\left(\mathbb{R}^{d}\right)\right)^{d}$ in the space of functions of subexponential growth at infinity. Moreover, according to [14], this solution is statistically homogeneous, $\mathbf{v}^{\kappa}(z, \omega)=\mathbf{v}^{\kappa}\left(T_{z} \omega\right)$, and the following a priori estimates hold

$$
\begin{aligned}
& \left|\mathbf{v}^{\kappa}(z, \omega)\right| \leqslant C \kappa^{-1}, \\
& \mathbf{E}\left\|\nabla \mathbf{v}^{\kappa}\right\|_{\left(L^{2}\left(S_{1}\right)\right)^{d^{2}}} \leqslant C ;
\end{aligned}
$$

here and in what follows $C$ stands for any generic nonrandom constant. We introduce a "volume-average" approximation of $\hat{a}$ on $S_{\rho}=[0, \rho]^{d}$ as follows

$$
\hat{a}^{\kappa, \rho}=\rho^{-d} \int_{S_{\rho}} a(z, \omega)\left(\nabla \mathbf{v}^{\kappa}(z, \omega)+I\right) d z .
$$

As was shown in [24], Lemma 2.5,

$$
\mathbf{E}\left|\hat{a}^{\kappa, \rho}-\hat{a}\right|^{2} \leqslant C\left(\kappa^{\beta}+\left(\kappa \rho^{2}\right)^{-\beta_{1}}\left(\log \left(\frac{1}{\kappa}\right)\right)^{\beta_{2}}\right),
$$

where $\beta, \beta_{1}, \beta_{2}$ and $C$ are strictly positive constants that only depend on the ellipticity constant $\lambda$, the exponent $\theta$ in (23), and the dimension $d$.

We proceed with the Approximation by Dirichlet problem, and consider an auxiliary Dirichlet problem

$$
\begin{aligned}
& -\operatorname{div}\left(a(z, \omega) \nabla \mathbf{w}^{\kappa, \rho}\right)+\kappa \mathbf{w}^{\kappa, \rho}=\operatorname{div} a(z, \omega) \text { in } S_{\rho}, \\
& \left.\mathbf{w}^{\kappa, \rho}\right|_{\partial S_{\rho}}=0 .
\end{aligned}
$$


We now use a solution $\mathbf{w}^{\kappa, \rho}$ to define

$$
\bar{a}^{\kappa, \rho}=\rho^{-d} \int_{S_{\rho}} a(z, \omega)\left(\nabla \mathbf{w}^{\kappa, \rho}(z, \omega)+I\right) d z .
$$

Then the difference $\left(\mathbf{v}^{\kappa}-\mathbf{w}^{\kappa, \rho}\right)$ satisfies the equation

$$
\begin{aligned}
& -\operatorname{div}\left(a(z, \omega) \nabla\left(\mathbf{v}^{\kappa}-\mathbf{w}^{\kappa, \rho}\right)\right)+\kappa\left(\mathbf{v}^{\kappa}-\mathbf{w}^{\kappa, \rho}\right)=0 \quad \text { in } S_{\rho}, \\
& \left.\left(\mathbf{v}^{\kappa}-\mathbf{w}^{\kappa, \rho}\right)\right|_{\partial S_{\rho}}=\left.\mathbf{v}^{\kappa}\right|_{\partial S_{\rho} .}
\end{aligned}
$$

We will estimate a solution of this equation separately in a smaller cube $\mathcal{S}_{\left(\rho-\rho^{\delta}\right)}=\left(\rho^{\delta}, \rho-\rho^{\delta}\right)^{d}$ with $0<\delta<1$, and in the boundary layer $S_{\rho} \backslash \mathcal{S}_{\left(\rho-\rho^{\delta}\right)}$.

In a slightly bigger cube $\left(\rho^{\delta}-1, \rho-\rho^{\delta}+1\right)^{d}$, by (25) and the bounds for the Green function of (31) given by Proposition 1 below, we obtain

$$
\left|\mathbf{v}^{\kappa}(z)-\mathbf{w}^{\kappa, \rho}(z)\right| \leqslant c \kappa^{-1} \exp \left(-C_{1} \sqrt{\kappa} \rho^{\delta}\right)
$$

with nonrandom constants $c$ and $C_{1}>0$. With the help of local elliptic estimates, based for instance on Corollary 8.7. in [10], this implies the inequality

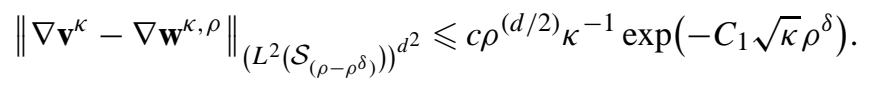

To estimate the contribution of the boundary layer $S_{\rho} \backslash \mathcal{S}_{\left(\rho-\rho^{\delta}\right)}$, we change the variables $x=z / \rho$ so that $S_{\rho}$ is transformed in $S_{1}$, and introduce the functions

$$
\mathbf{v}_{0}^{\kappa, \rho}(x, \omega)=\frac{1}{\rho} \mathbf{v}^{\kappa}(\rho x, \omega) ; \quad \mathbf{w}_{0}^{\kappa, \rho}(x, \omega)=\frac{1}{\rho} \mathbf{w}^{\kappa, \rho}(\rho x, \omega) .
$$

These functions satisfy in $S_{1}$ the equations

$$
\begin{aligned}
& -\operatorname{div}\left(a(\rho x, \omega) \nabla \mathbf{v}_{0}^{\kappa, \rho}+\rho^{2} \kappa \mathbf{v}_{0}^{\kappa, \rho}=\operatorname{div} a(\rho x, \omega),\right. \\
& -\operatorname{div}\left(a(\rho x, \omega) \nabla \mathbf{w}_{0}^{\kappa, \rho}+\rho^{2} \kappa \mathbf{w}_{0}^{\kappa, \rho}=\operatorname{div} a(\rho x, \omega),\right.
\end{aligned}
$$

and formula (27) and (30) read respectively

$$
\begin{aligned}
\hat{a}^{\kappa, \rho} & =\int_{S_{1}} a(\rho x, \omega)\left(\nabla \mathbf{v}_{0}^{\kappa, \rho}(x, \omega)+I\right) d x, \\
\bar{a}^{\kappa, \rho} & =\int_{S_{1}} a(\rho x, \omega)\left(\nabla \mathbf{w}_{0}^{\kappa, \rho}(x, \omega)+I\right) d x .
\end{aligned}
$$

Standard energy estimates in (35) yield

$$
\left\|\nabla \mathbf{w}_{0}^{\kappa, \rho}\right\|_{\left(L^{2}\left(S_{1}\right)\right)^{d^{2}}} \leqslant C .
$$

Using the notation $\Pi(\rho, \delta)$ for the boundary layer $S_{1} \backslash\left(\rho^{\delta-1}, 1-\rho^{\delta-1}\right)^{d}$, and considering (25), (33) and the last estimate, we get

$$
\begin{aligned}
\mathbf{E}\left|\bar{a}^{\kappa, \rho}-\hat{a}^{\kappa, \rho}\right|^{2} & =\mathbf{E}\left(\int_{S_{1}} a(\rho x, \omega)\left(\nabla \mathbf{w}_{0}^{\kappa, \rho}-\nabla \mathbf{v}_{0}^{\kappa, \rho}\right) d x\right)^{2} \\
& \leqslant 3 \mathbf{E}\left(\int_{\rho^{-1} \mathcal{S}_{\left(\rho-\rho^{\delta}\right)}} a(\rho x, \omega)\left(\nabla \mathbf{w}_{0}^{\kappa, \rho}-\nabla \mathbf{v}_{0}^{\kappa, \rho}\right) d x\right)^{2}
\end{aligned}
$$




$$
\begin{aligned}
& +3 \mathbf{E}\left(\int_{\Pi(\rho, \delta)} a(\rho x, \omega) \nabla \mathbf{w}_{0}^{\kappa, \rho} d x\right)^{2}+3 \mathbf{E}\left(\int_{\Pi(\rho, \delta)} a(\rho x, \omega) \nabla \mathbf{v}_{0}^{\kappa, \rho} d x\right)^{2} \\
\leqslant & C \kappa^{-1} \exp \left(-C_{1} \sqrt{\kappa} \rho^{\delta}\right)+C \mathbf{E}\left(\int_{\Pi(\rho, \delta)}\left|\nabla \mathbf{w}_{0}^{\kappa, \rho}\right|^{2} d x \int_{\Pi(\rho, \delta)} 1 d x\right) \\
& +C \mathbf{E}\left(\int_{\Pi(\rho, \delta)}\left|\nabla \mathbf{v}_{0}^{\kappa, \rho}\right|^{2} d x \int_{\Pi(\rho, \delta)} 1 d x\right) \\
\leqslant & C \kappa^{-1} \exp \left(-C_{1} \sqrt{\kappa} \rho^{\delta}\right)+C \rho^{\delta-1}+C \rho^{\delta-1} .
\end{aligned}
$$

We summarize this in the following

Lemma 1. Let $\hat{a}^{\kappa, \rho}$ and $\bar{a}^{\kappa, \rho}$ be the matrices defined in (27) and (30), respectively. Then, the difference $\left(\bar{a}^{\kappa, \rho}-\hat{a}^{\kappa, \rho}\right)$ admits the bound

$$
\mathbf{E}\left|\bar{a}^{\kappa, \rho}-\hat{a}^{\kappa, \rho}\right|^{2} \leqslant C \kappa^{-1} \exp \left(-C_{1} \sqrt{\kappa} \rho^{\delta}\right)+C \rho^{\delta-1} .
$$

Our next step is to estimate the difference between $\bar{a}^{\kappa, \rho}$ and $\bar{a}^{\rho}$ defined in (30) and (13) respectively. To this end we rewrite Eq. (21) as follows

$$
\begin{aligned}
& -\operatorname{div}\left(a(\rho x, \omega) \nabla \mathbf{w}_{0}^{\rho}\right)=\operatorname{div} a(\rho x, \omega) \text { in } S_{1}, \\
& \left.\mathbf{w}_{0}^{\rho}\right|_{\partial S_{1}}=0 .
\end{aligned}
$$

Equivalently, this equation can be written in the form

$$
-\operatorname{div}\left(a(\rho x, \omega) \nabla\left(\mathbf{w}_{0}^{\rho}+x\right)\right)=0 .
$$

This equation is a particular case of problem (4); thus it can be homogenized in a standard way, and $\mathbf{w}_{0}^{\rho}$ converges, as $\rho \rightarrow \infty$, to a solution of the effective problem

$$
-\operatorname{div}\left(\hat{a} \nabla\left(\mathbf{w}_{0}^{\infty}+x\right)\right)=0,\left.\quad \mathbf{w}_{0}^{\infty}\right|_{\partial S_{1}}=0 .
$$

Clearly, $\mathbf{w}_{0}^{\infty} \equiv 0$, and according to [24], Theorem 3.1,

$$
\mathbf{E}\left\|\mathbf{w}_{0}^{\rho}\right\|_{\left(L^{2}\left(S_{1}\right)\right)^{d}}^{2}=\mathbf{E}\left\|\mathbf{w}_{0}^{\rho}-\mathbf{w}_{0}^{\infty}\right\|_{\left(L^{2}\left(S_{1}\right)\right)^{d}}^{2} \leqslant C \rho^{-\beta} .
$$

Subtracting (38) from the second equation in (35) gives

$$
-\operatorname{div}\left(a(\rho x, \omega) \nabla\left(\mathbf{w}_{0}^{\kappa, \rho}-\mathbf{w}_{0}^{\rho}\right)\right)+\rho^{2} \kappa\left(\mathbf{w}_{0}^{\kappa, \rho}-\mathbf{w}_{0}^{\rho}\right)=-\rho^{2} \kappa \mathbf{w}_{0}^{\rho} .
$$

In view of (39), an energy estimate yields

$$
\mathbf{E}\left\|\nabla \mathbf{w}_{0}^{\kappa, \rho}-\nabla \mathbf{w}_{0}^{\rho}\right\|_{\left(L^{2}\left(S_{1}\right) d^{d^{2}}\right.}^{2} \leqslant C \rho^{2-\beta} \kappa .
$$

Therefore, with the definitions (37) and (13) we have

$$
\mathbf{E}\left|\bar{a}^{\kappa, \rho}-\bar{a}^{\rho}\right|^{2} \leqslant C \rho^{2-\beta} \kappa .
$$

Now, combining (28), (41), and the estimate of Lemma 1, we arrive at the inequality

$$
\mathbf{E}\left|\hat{a}-\bar{a}^{\rho}\right|^{2} \leqslant C\left(\left[\kappa^{\beta}+\left(\kappa \rho^{2}\right)^{-\beta_{1}}\left(\log \frac{1}{\kappa}\right)^{\beta_{2}}\right]+\left[\kappa^{-1} \exp \left(-C_{1} \sqrt{\kappa} \rho^{\delta}\right)+\rho^{\delta-1}\right]+\rho^{2-\beta} \kappa\right) .
$$

It remains to select $\kappa$ and $\delta$. If we set $\kappa=\rho^{\gamma-2}$ with sufficiently small $\gamma>0$, then

$$
\mathbf{E}\left|\hat{a}-\bar{a}^{\rho}\right|^{2} \leqslant C\left(\left[\rho^{-\beta(2-\gamma)}+\rho^{-\gamma \beta_{1}} \log \rho\right]+\left[\rho^{2-\gamma} \exp \left(-C_{1} \rho^{\frac{\gamma}{2}-1+\delta}\right)+\rho^{\delta-1}\right]+\rho^{\gamma-\beta}\right) .
$$


Letting $\gamma=\frac{\beta}{2}$ and $\delta=1-\frac{\beta}{8}$, we obtain

$$
\mathbf{E}\left|\hat{a}-\bar{a}^{\rho}\right|^{2} \leqslant C\left(\left[\rho^{-\beta\left(2-\frac{\beta}{2}\right)}+\rho^{-\frac{\beta}{2} \beta_{1}} \log \rho\right]+\left[\rho^{2-\frac{\beta}{2}} \exp \left(-C_{1} \rho^{\frac{\beta}{8}}\right)+\rho^{-\frac{\beta}{8}}\right]+\rho^{-\frac{\beta}{8}}\right) .
$$

Finally, the following statement holds

Theorem 4. Under the mixing condition (23), the difference between the homogenized matrix $\hat{a}$ given by (7) and its approximation $\bar{a}^{\rho}$ given by (13), satisfies the estimate

$$
\mathbf{E}\left|\hat{a}-\bar{a}^{\rho}\right|^{2} \leqslant C \rho^{-\beta_{3}}, \quad \beta_{3}>0,
$$

with $\beta_{3}=\beta_{3}(\theta, \lambda, d)$.

To make the proof of Theorem 4 complete, we proceed now to the Green function estimate used in (32). Let $A=\frac{\partial}{\partial x_{i}} a_{i j}(x) \frac{\partial}{\partial x_{j}}+c(x)$ be a uniformly elliptic operator in $\mathbb{R}^{n}$ such that

$$
\Lambda^{-1} I \leqslant a(x) \leqslant \Lambda I, \quad c(x) \leqslant-\mu, \mu>0 .
$$

In a bounded domain $Q \subset \mathbb{R}^{n}$ consider a Dirichlet problem

$$
A u=0,\left.\quad u\right|_{\partial Q}=\phi(x) .
$$

The proof of the following result can be found in [16], p. 61.

Proposition 1. Suppose that $0 \leqslant \phi(x) \leqslant 1$ for all $x \in \partial Q$. Then the solution $u(x)$ of problem (42) satisfies the estimate

$$
u(x) \leqslant c_{0} \exp \left(-c_{1} \sqrt{\mu} \operatorname{dist}(x, \partial Q)\right),
$$

with constants $c_{0}>0$ and $c_{1}>0$ only depending on $\Lambda$ and $n$.

The other two models introduced in Section 1, namely Periodic Approximation and Approximation by Neumann problem, can be studied in a similar way. We proceed with a brief description of the results for the periodic approximation. In this case the following statement holds

Theorem 5. Under the mixing condition (23) the discrepancy $\left(\hat{a}-\tilde{a}^{\rho}\right)$ with $\hat{a}$ given by (7) and its approximation $\tilde{a}^{\rho}$ given by (10), satisfies the estimate

$$
\mathbf{E}\left|\hat{a}-\tilde{a}^{\rho}\right|^{2} \leqslant C \rho^{-\beta_{3}}, \quad \beta_{3}>0,
$$

with $\beta_{3}=\beta_{3}(\theta, \lambda, d)$.

We give only a sketch of the proof. We introduce a function $\chi^{\kappa, \rho}$ being a $S_{\rho}$-periodic solution of the "penalized" equation

$$
-\operatorname{div}\left(a(z, \omega) \nabla \chi^{\kappa, \rho}\right)+\kappa \chi^{\kappa, \rho}=\operatorname{div} a(z, \omega),
$$

and define an intermediate approximation of effective coefficients by

$$
\tilde{a}^{\kappa, \rho}=\rho^{-d} \int_{S_{\rho}} a(z, \omega)\left(\nabla \chi^{\kappa, \rho}(z, \omega)+I\right) d z .
$$

The estimates for the fundamental solution of (44) imply the bound

$$
\left|\chi^{\kappa, \rho}(z, \omega)\right| \leqslant C \kappa^{-1}
$$


with a nonrandom constant $C$. Denote $\chi_{0}^{\kappa, \rho}(x, \omega)=\frac{1}{\rho} \chi^{\kappa, \rho}(\rho x, \omega)$. In the same way as above one can estimate the difference of matrices $\hat{a}^{\kappa, \rho}$ and $\tilde{a}^{\kappa, \rho}$ defined in (27) and (45) respectively:

Lemma 2. Let $\hat{a}^{\kappa, \rho}$ and $\tilde{a}^{\kappa, \rho}$ be the matrices defined in (27) and (45), respectively. Then, the difference $\left(\hat{a}^{\kappa, \rho}-\tilde{a}^{\kappa, \rho}\right)$ admits the bound

$$
\mathbf{E}\left|\hat{a}^{\kappa, \rho}-\tilde{a}^{\kappa, \rho}\right|^{2} \leqslant C\left(\kappa^{-1} \exp \left(-C_{1} \sqrt{\kappa} \rho^{\delta}\right)+\rho^{\delta-1}\right) .
$$

Then we compare $\chi_{0}^{\kappa, \rho}$ and a $S_{1}$-periodic solution of the equation

$$
-\operatorname{div}\left(a(\rho x, \omega)\left(\nabla \chi_{0}^{\rho}+I\right)\right)=0,
$$

which is just a vector form of Eq. (17). As was shown in the proof of Theorem $1, \chi_{0}^{\rho}$ converges to $\chi_{0}^{\infty} \equiv 0$ in $\left(L^{2}\left(S_{1}\right)\right)^{d}$, as $\rho \rightarrow \infty$. Moreover, the analysis of proof of Theorem 3.1 in [24] shows that the statement of this theorem remains valid for a problem with periodic boundary condition instead of Dirichlet condition. Therefore,

$$
\mathbf{E}\left\|\chi_{0}^{\rho}\right\|_{\left(L^{2}\left(S_{1}\right)\right)^{d}}^{2} \leqslant C \rho^{-\beta}
$$

with $\beta>0$. The rest of the proof or Theorem 5 is just the same as that of Theorem 4 , and we skip the details.

Similar result also holds for approximation by means of Neumann problem.

Theorem 6. Under mixing condition (23) the discrepancy between the homogenized matrix $\hat{a}$ given by (7) and its Neumann problem approximation $\check{a}^{\rho}$ given by (15), satisfies the estimate

$$
\mathbf{E}\left|\hat{a}-\check{a}^{\rho}\right|^{2} \leqslant C \rho^{-\beta_{3}}, \quad \beta_{3}>0,
$$

with $\beta_{3}=\beta_{3}(\theta, \lambda, d)$.

Remark 2. In order to construct the Dirichlet or Neumann problem Approximations one can deal with homothetic dilatations of a regular bounded domain instead of the cubic samples used herein. In addition, any mixed boundary condition could have been used for the truncated problem, instead of pure Dirichlet or Neumann boundary conditions, as soon as these boundary conditions are satisfied by the function $\eta \cdot z$. In all those cases the techniques of this work also apply and similar convergence results and error bounds would have been obtained.

\section{Acknowledgements}

The work of both authors has been partially supported by grant from the CNRS/ANDRA/BRGM/CEA/EDF GDR 2439 whose support is gratefully acknowledged. Also, the work of A. Piatnitski has been partially supported by RFBR, grants 00-01-22000 and 02-01-00868, and by INTAS, grant 99-559.

\section{References}

[1] A. Badea, A. Bourgeat, Modeling and Computation in Environmental Sciences, in: Notes in Numerical Fluids Mechanics, vol. 59, 1997, pp. 13-24.

[2] J. Bear, Dynamics of Fluids in Porous Media, Elsevier, New York, 1972.

[3] G. Dal Maso, L. Modica, Nonlinear stochastic homogenization and ergodic theory, J. Reine Angew. Math. 368 (1986) $28-42$.

[4] A. Bensoussan, J.-L. Lions, G. Papanicolaou, Asymptotic Analysis of Periodic Structures, North Holland, Amsterdam, 1978.

[5] A. Bourgeat, M. Quintard, S. Whitaker, Eléments de comparaison entre la méthode d'homogénéisation et la méthode de prise de moyenne avec fermeture, C. R. Acad. Sci. Paris Sér. II 306 (1988) 463-466.

[6] A. Bourgeat, A. Mikelic, S. Wright, Stochastic two-scale convergence in the mean and applications, J. Reine Angew. Math. 456 (1994) $19-51$. 
[7] A. Bourgeat, S.M. Kozlov, A. Mikelić, Effective equations of two-phase flow in random media, Cal. Var. PDE 8 (1998) 1-31.

[8] N. Dunford, J.T. Schwartz, Linear Operators, Part I, General Theory, Interscience, 1963.

[9] L.J. Durlofski, Representation of grid block permeability in coarse scale models of randomly heterogeneous porous media, Water Resources Res. 28 (1992) 1791-1800.

[10] D. Gilbarg, N.S. Trudinger, Elliptic Partial Differential Equations of Second Order, Springer-Verlag, 1977.

[11] R. Hill, Elastic properties of reinforced solids: some theoretical principles, J. Mech. Phys. Solids 11 (1963) 357-372.

[12] U. Hornung (Ed.), Homogenization and Porous Media, IAM, vol. 6, Springer-Verlag, Berlin, 1997.

[13] V.V. Jikov, S.M. Kozlov, O.A. Oleinik, Homogenization of Differential Operators and Integral Functionals, Springer-Verlag, New York, 1994.

[14] S.M. Kozlov, The averaging of random operators, Math. USSR Sb. 109 (1979) 188-202.

[15] S.M. Kozlov, Geometric aspects of homogenization, Russian Math. Surv. 44 (1989) 91-144.

[16] N. Krylov, Controlled Diffusion Processes, in: Application of Mathematics, vol. 14, Springer-Verlag, New York, 1980.

[17] T. Lewinski, J. Telega, Plates, Laminates and Shells. Asymptotic Analysis and Homogenization, in: Series on Advances in Mathematics for Applied Sciences, vol. 52, World Scientific, River Edge, NJ, 2000.

[18] L. Modica, Stochastic Homogenization and Ergodic Theory, in: Conti, De Giorgi, Gianessi (Eds.), Proceedings ERICE 1984, in: Lecture Notes in Mathematics, vol. 1190, Springer-Verlag, 1986, Chapter 15.

[19] H. Owhadi, Approximation of effective conductivity of ergodic media by periodization, Probab. Theory Related Fields 125 (2003) 225 258.

[20] G. Papanicolaou, S.R.S. Varadhan, Boundary value problems with rapidly oscillating random coefficients. Random fields. Rigorous results in statistical mechanics and quantum field theory, Esztergom 1979, Colloq. Math. Soc. Janos Bolyai 27 (1981) $835-873$.

[21] K. Sab, On the homogenization and the simulation of random materials, Eur. J. Mech., A/Solids 11 (5) (1992) $585-607$.

[22] A.V. Pozhidaev, V.V. Yurinskii, On the error of averaging of symmetric elliptic systems, Math. USSR-Izv. 35 (1) (1990) 183-201.

[23] Ph. Renard, G. DeMarsily, Calculating equivalent permeability: A review, Adv. Water Resour. 20 (1997) $253-278$.

[24] V.V. Yurinski, Averaging of symmetric diffusion in random medium, Sibirskii Mat. Zh. 27 (4) (1986) $167-180$. 